\title{
Annexe : Le Maroc
}

\section{(2) OpenEdition \\ 1 Journals}

Édition électronique

URL : http://journals.openedition.org/conflits/191

DOI : 10.4000/conflits. 191

ISSN : $1777-5345$

Éditeur :

CCLS - Centre d'études sur les conflits lilberté et sécurité, L'Harmattan

Édition imprimée

Date de publication : 15 mai 1994

ISSN : 1157-996X

Référence électronique

"Annexe : Le Maroc », Cultures \& Conflits [En ligne], 13-14 I printemps-été 1994, mis en ligne le, consulté le 30 mars 2021. URL : http://journals.openedition.org/conflits/191 ; DOI : https://doi.org/ 10.4000/conflits. 191

Ce document a été généré automatiquement le 30 mars 2021.

Creative Commons License 


\section{Annexe : Le Maroc}

Le problème sahraouis Il est difficile de parler des "disparus" sahraouis, car au Maroc personne n'osait et n'ose, même encore maintenant, véritablement l'aborder. La fameuse "marche verte" en 1975 a endormi les consciences des Marocains qui se réfèrent toujours à l'interdiction de porter atteinte à l'intégrité territoriale. On peut observer qu'encore le 30 juin dernier, les partis d'opposition n'étaient toujours pas prêts à reconnaître une éventuelle indépendance de la République sahraouie puisqu'on pouvait voir dans une information de l'AFP que "quatre partis marocains d'opposition ont adressé au Président égyptien, M. Hosni Moubarak, nouveau président de l'OUA, un message lui demandant de suspendre l'adhésion de la prétendue République sahraouie de l'organisation panafricaine...." "celle-ci ne disposant au Sahara Occidental d'aucune entité territoriale ou populaire pas plus que de légalité internationale. Et pourtant, Amnesty a estimé qu'entre 1975 et 1985, plusieurs centaines de personnes du Sud marocain et du Sahara occidental ont été arrêtées par des forces de sécurité et ont disparu dans des centres secrets. Bien que les Sahraouis instruits aient été plutôt la cible des "disparitions", des vieillards et de tous jeunes enfants figurent au nombre des victimes. Même des familles entières "disparaissaient". Presque tous les sahraouis maintenus en détention prolongée sans jugement pour des motifs politiques n'ont jamais bénéficié des procédures légales normales. Dans tous les cas, les informations recueillies laissent à penser que les autorités marocaines soupçonnaient les victimes ou leurs familles de soutenir le Front Polisario. C'est ainsi que Ahmed Lemaadal Mohamed Medhi El Bou, originaire de Smara, une des principales villes du Sahara occidental, a disparu à 33 ans, après avoir été arrêté le 15 avril 1976 au cours d'une rafle de civils sahraouis soupçonnés de soutenir le Front Polisario. Il avait servi dans l'armée espagnole, et comme la plupart des autres militaires d'origine sahraouie, avait été rendu à la vie civile à la veille de l'invasion de la colonie espagnole par le Maroc. Sa femme et ses trois fils sont sans nouvelles de lui. Ils ont fui le Sahara occidental pour rejoindre les milliers de réfugiés sahraouis des camps du Front Polisario dans le sud de l'Algérie. Ainsi le Sahraoui Kenti Sidi Balla peut témoigner de ce qu'il a vécu : "Je fus arrêté le 19 juin 1987 par des soldats marocains... ils m'ont remis à la gendarmerie royale... là j'ai été torturé et interrogé pendant 24 heures... j'ai été transféré dans un autre centre de détention à Skoura où il $\mathrm{y}$ avait quatorze autre Sahraouis dont des 
femmes... Au bout de deux ans j'ai été transféré au centre secret de Kalaat M'Gouna. Il fut libéré en août 1991.

Arbitraire et vengeance La multiplicité des systèmes policiers fait qu'il était souvent difficile de savoir pourquoi certains opposants plutôt que d'autres "disparaissaient". Le choix pouvait être totalement arbitraire. Mohamed Nadrani en est l'exemple type. En 1976 Mohamed Nadrani avait 22 ans, il était étudiant et était syndiqué comme beaucoup d'autres étudiants. Certains de ses camarades ont été arrêtés et emprisonnés, d'autres relâchés, lui a été maintenu en détention secrète pendant neuf ans, dont dix huit mois à l'isolement total. Après sa libération, il a demandé à consulter son casier judiciaire. Il était vierge. Officiellement, Mohamed Nadrani n'a jamais été détenu. Cependant, les "disparitions" étaient aussi dues à des vengeances personnelles. Une personne commettant un délit pouvait provoquer la "disparition" d'un membre de la famille ou de la famille tout entière. Donnons deux exemples pour illustrer ce type de "disparition".

La famille Oufkir Huit membres de la famille ont été détenus pendant 18 ans, simplement à cause de leurs liens familiaux avec le général Mohamed Oufkir, décédé dans des circonstances mystérieuses après qu'il ait mené un coup d'Etat contre le roi. Sa veuve, ses six enfants, dont le plus jeune n'avait que 3 ans, ainsi qu'une cousine avaient subitement disparus. Les autorités marocaines ne fournissaient aucune information sur leur sort et leur lieu de détention, comme s'ils n'avaient jamais existé. C'est parce qu'en 1987, quatre enfants ont réussi à s'évader, rencontré un avocat français qui a alerté l'opinion publique que la famille sera en fin libérée en 1991. Le pouvoir s'acharne encore sur cette famille innocente, puisqu'elle reste interdite de passeport, malgré une pression nationale et internationale importante.

Les militaires de Tazmamart Cinquante huit militaires qui avaient participé à un attentat contre le roi et qui avaient été jugés et condamnés, pour la plupart, à des peines allant de 3 ans à 10 ans de prison, ont été transférés de la prison centrale de Kénitra au centre secret de Tazmamart, dans les contreforts de l'atlas. Ce centre avait été spécialement construit pour eux. Les cinquante huit cellules n'avaient ni fenêtre, ni éclairage, suffocantes l'été et glaciales l'hiver. Pendant 18 ans, ils ne sont jamais sortis de leurs cellules, n'ont jamais vu le soleil et n'ont jamais reçu aucun soin médical. Deux ans après leur arrivée à Tazmamart, deux d'entre eux terminèrent de purger leur peine. Voyant qu'ils n'étaient pas libérés, comme ils s'y attendaient, l'un d'eux se plaignit au gardien : "Tu en avais pour combien ?" il répondit "Trois ans". Le gardien rétorque : "Ne dis pas trois ans, dis la perpétuité". Vingt huit prisonniers moururent à Tazmamart. Seuls trente d'entre eux furent libérés en 1991.

La raison d'Etat, l'opposition politique au régime n'étaient pas les seules causes de "disparitions". Il pouvait aussi y avoir des jalousies privées, des rivalités personnelles ou des opérations financières secrètes. C'est ainsi que l'on peut citer le cas incroyable des trois frères Bourequat, de nationalité française et proche du Makzen (pouvoir royal). Ils furent enfermés de 1973 à 1991, dont les dix dernières années à Tazmamart, sans inculpation, ni jugement. Dans leurs témoignages, ils ont affirmé que "leur affaire avait commencé par la dénonciation au roi, sa majesté Hassan II, d'une conspiration dirigée par le chef des services secrets marocains, le colonel Dlimi".

En finir avec les disparus A l'heure actuelle, bien qu'il y ait encore environ 80 "disparus" marocains et 485 "disparus" au Sahara occidental, on ne disparait plus ou presque plus au Maroc. La diminution du nombre des "disparitions" signalées depuis 
1987 peut être due à l'amélioration du respect des limites légales de la garde à vue. En effet, le 30 décembre 1991, le roi Hassan II a promulgué une nouvelle loi amendant certains articles du code de procédure pénale marocain relatifs à la garde à vue (période pendant laquelle la personne interpellée est retenue par la police sans pouvoir entrer en contact avec sa famille ou un avocat).

Cependant d'autres raisons peuvent être avancées :

- Il semble que le pouvoir se sente plutôt embarrassé, en fait, on pourrait même dire pris à son propre piège. Il nie un jour, libère le lendemain, exige qu'on n'en parle plus, menace même. Il tergiverse et se rend à l'évidence malgré lui. Rappelons-nous : . En novembre 1990, le Comité des Droits de l'Homme des Nations Unies ayant soulevé enfin le problème de Tazmamart, les représentants marocains avaient alors déclaré que le nom de Tazmamart ne figurait sur aucune des listes officielles des prisons. . En juillet 1991, le roi Hassan II affirmait publiquement que "Tazmamart n'existait que dans l'esprit et l'imagination de personnes mal intentionnées". . Un mois plus tard, les 28 militaires qui avaient survécu sortaient de leur bagne-mouroir. . Et ce n'était qu'en 1992 que le roi reconnaissait l'existence de Tazmamart. Il déclarait alors pour le journal Libération qu'"il s'agissait d'un lieu où ont été gardées des personnes qui y ont été administrativement assignées. Il n'a plus de raison d'être. Ce chapitre est clos. Il y a eu. Il n'y a plus. C'est tout".

- Autre raison : le livre de Gilles Perrault "Notre ami le roi", qui avait fait grand bruit et peut-être atteint quelques consciences endormies, bien que le pouvoir ait essayé d'en arrêter la diffusion au Maroc.

- N'oublions pas aussi le travail incessant de nombreuses associations de droits de l'homme en dehors du Maroc ainsi bien sûr qu'une grande campagne au niveau international organisée par Amnesty en 1991. Toutes ces manifestations ont eu pour effet, en 1991, la libération de plus de 260 Sahraouis, de 28 militaires de Tazmamart, des trois frères Bourequat et de la famille Oufkir. Bien que, depuis 1991, on soit obligé de constater, qu'au strict point de vue des libérations de "disparus", nous n'avons obtenu aucun résultat, cependant nous pouvons dire que maintenant le Maroc est un pays avec des forces intérieures puissantes demandant le changement et avec un gouvernement sensible à son image et aux pressions extérieures. En effet :

- Les journaux d'opposition n'hésitent plus à dénoncer les arrestations et les jugements inéquitables et surtout, ce qui est nouveau, demandent avec insistance que tous les "disparus soient libérés". Ils osent publier des lettres ouvertes d'anciens prisonniers de Tazmamart qui demandent réparation aux autorités. Et même, ils font paraître des interview de familles de disparus comme celle par exemple des parents de Houcine el Manouzi : Ce syndicaliste avait 28 ans lorsqu'il a été condamné à mort par contumace, à Marrakech, en septembre 1971, il a été enlevé à Tunis, l'année suivante, le 28 octobre 1972, et ramené au Maroc. Selon les observateurs d'Amnesty International, le procès avait été manifestement inéquitable, la majorité des accusés ne pouvant avoir commis les crimes qui leur étaient reprochés. Depuis 21 ans sa famille n'a rien pu savoir de lui, sinon qu'il a tenté de s'évader d'un lieu de détention avec d'autres "disparus". Il semble avoir été repris une semaine plus tard. Sa famille habite Paris et a créé l'Association des Parents et Amis des Disparus au Maroc.

- Et puis maintenant les langues se délient. Les anciens "disparus" crient bien haut qu'il faut parler, qu'il faut témoigner, que le mur du silence doit tomber.

- L'Association des Familles des Disparus et des Prisonniers politiques s'est mobilisée le 
10 décembre dernier, à l'occasion du 45ème anniversaire de la Déclaration Universelle des Droits de l'Homme, pour demander la libération de tous les "disparus" et les prisonniers politiques. Ce même jour, deux organisations des Droits de l'Homme exigeaient que la lumière soit faite sur les prisonniers politiques "disparus". Elles n'hésitent plus à faire des "disparitions" une priorité avec la publication de liste de "disparus". Vient s'ajouter la création de comités dans quarante villes du Maroc. Ces comités ont l'intention de faire de 1994 une année spécialement réservée aux disparitions et cette fois-ci Marocains et Sahraouis seront concernés. Quant au pouvoir, nous pouvons avoir quelques espoirs, même s'ils restent un peu mitigés :

- Amnesty International a pu se rendre à deux reprises au Maroc en 1993, ce qui n'était pas arrivé depuis 1990. Elle a même pu avoir des rencontres intéressantes avec des interlocuteurs concernés par les problèmes des Droits de l'Homme.

- En décembre dernier le Ministre de l'Intérieur et le Conseil Consultatif des Droits de l'Homme (créé par le roi) ont tous deux déclaré qu'ils étaient déterminés à liquider tous les dossiers relatifs au Droits de l'Homme. Néanmoins ils démentaient catégoriquement, une fois de plus, l'existence de centres secrets et donnaient l'assurance que des certificats de décès seraient envoyés aux familles. A ce sujet, nous ne savons que penser car, à ce jour, nous n'avons aucune nouvelle des 600 "disparus" pour lesquels nous avons remis une liste aux autorités marocaines.

- Un Ministre délégué auprès du Premier Ministre, chargé des Droits de l'Homme, a été nommé dans le nouveau gouvernement. Bien sûr, nous pouvons nous interroger : "A-til le désir d'agir et le laissera-t-on faire ou est-ce une nouvelle façade et un comportement caricatural du pouvoir marocain vis-à-vis des ses engagements internationaux? A l'extérieur, malgré quelques interventions du Département d'Etat américain ou du Parlement Européen, dénonçant même fermement les violations des Droits de l'Homme, le Maroc donne l'image d'un pays stable et sûr à l'étranger, comparé à la situation actuelle en Algérie. Ceci est notamment dû aux bonnes relations économiques avec les pays occidentaux y compris ceux d'Amérique du Nord.

\section{INDEX}

Mots-clés : disparitions

Index géographique : Maghreb, Maroc 